\title{
Congenital Heart Disease Revealing Familial 22q11 Deletion Syndrome
}

Marlene Viviane Pires Fernandes Santos, ${ }^{\circledR}$ Bruno Faulin Gamba, ${ }^{\circledR}$ Stefany Lucas Lopes Empke, ${ }^{2}$ Camila Cristina de Oliveira Alves, ${ }^{2 \oplus}$ Nádia Aparecida Bérgamo, ${ }^{1 \oplus}$ Lucilene Arilho Ribeiro-Bicudo $^{1 \oplus}$

Universidade Federal de Goiás - Campus Samambaia,' Goiás, GO - Brazil

Universidade Estadual Paulista - Campus de Botucatu, ${ }^{2}$ São Paulo, SP - Brazil

\section{Abstract}

Congenital heart defects are the most common birth defects and the leading cause of mortality in the first year of life. It is well known that the 22q11 deletion syndrome (22q11DS) is the most common microdeletion syndrome in humans and that congenial heart diseases (CHDs) are one of the most common phenotypic manifestations. However, it should be noted that the 22q11 deletion was also found in a significant number of patients with isolated CHD. The 22q11DS phenotype may include cardiovascular anomalies, palatal abnormalities, nasal voice, immune deficiency, endocrine dysfunctions, a varying degree of cognitive deficits and intellectual disabilities, velopharyngeal insufficiency, and characteristic craniofacial dysmorphism. This condition affects about 1 in 4,000 live births, making 22q11DS the most common microdeletion syndrome in humans. Here we describe the cases of three children who were referred to the clinical hospital center with the diagnosis of CHD, but with no direct signs of 22q11DS. Investigation of familial data led us to suspect that the mothers could be carriers of 22q11DS. The multiplex ligation-dependent probe amplification (MLPA) testing confirmed that the patients and mothers exhibited $3 \mathrm{Mb}$ 22q11 deletions, which justified the clinical signs in the mothers and the CHD in children. In the presence of a few characteristics that are common of a spectrum of some known syndromes, a familial examination can provide

\section{Keywords}

Congenital Heart Disease/genetics; Face/abnormalities/ genetics; DiGeorge Syndrome/genetics; Chromosomes, Human, Pair 22/genetics; Chromosome Deletion. clues to a definitive diagnosis, as well as to the prevention of diseases and genetic counseling of these patients.

\section{Introduction}

Congenital heart defects (CHDs) are the most common group of birth defects in humans that arise during cardiac embryogenesis and differ in morphology, physiology, and clinical outcome. They occur in about $1 \%$ of all live births irrespective of ethnic backgrounds, socioeconomic conditions, and geographic barriers. ${ }^{1}$ The causes of CHDs are multiple, and typically categorized in genetic and nongenetic factors. Nongenetic factors include teratogenic exposures during pregnancy and epigenetic alterations. Identifiable genetic etiologies are reported to be as high as $40 \%$ in syndromic CHD, including single gene disorders, chromosomal anomalies, and copy number variations $(\mathrm{CNV}){ }^{2}$

The 22q11.2 deletion syndrome (22q11DS; Online Mendelian Inheritance in Man \#602054) is the most frequent microdeletion syndrome, and is one of the most common genetic causes of CHD, responsible for $1.5 \%$ to $5 \%$ of all CHD at birth. ${ }^{3}$ This genetic disorder affects pharyngeal and neurobehavioral development, and causes congenital heart defects, velopharyngeal insufficiency, hypoparathyroidism, thymic aplasia or hypoplasia, craniofacial dysmorphism, learning difficulties, and psychiatric disorders. ${ }^{1}$ Combination of these signs differ from patient to patient, resulting in a large number of phenotypes ranging from normal to severely handicapped individuals. ${ }^{2}$ The frequency of clinical signs was previously reported by our group in a study involving 179 patients with 22q11DS confirmed by genetic tests (Table 1). Usually, $15-20 \%$ of the CHDs are conotruncal heart defects (CTDs). CTDs are more commonly associated with 22q11DS and comprise

Mailing Address: Bruno Gamba

Avenida Esperança, S/N. Postal Code: 74001-970, Goiânia, Goiás, GO - Brazil.

E-mail: gamba.bf@hotmail.com 
structural malformations that are similar to ventricular outflow tract defects. These include the Tetralogy of Fallot (TOF), pulmonary atresia with ventricular septal defect (PA-VSD), a double outlet right ventricle, transposition of the great arteries, persistent truncus arteriosus, and an interrupted aortic arch. ${ }^{3}$ Low-copy repeats (LCRs) of 22q11 have been suggested to mediate a non-allelic homologous recombination, resulting in $22 q$ rearrangement. An unequal crossover between the LCRs usually results in a 3 mebabase $(\mathrm{Mb})$ deletion in one copy of chromosome 22, and a reciprocal and similarly sized duplication in the other one. ${ }^{4} \mathrm{~A}$ microscopic deletion at 22q11 occurs in approximately 1 out every 4,000 live births. Different deletion genotypes have been delineated to this condition: a predominant $3-\mathrm{Mb}$ deletion accounting for $90 \%$ of the cases, a $1.5-2-\mathrm{Mb}$ deletion in $8 \%$, and atypical smaller deletions in $2 \%$. Phenotypic variability has been attributed to the presence or absence of genes in the breaking points, and CNVs depending on the size of the deletion. ${ }^{5}$ Familial cases of 22q11DS present a higher frequency of uncommon 1.5-2 Mb deletions; ${ }^{6}$ patients with this deletion have symptoms that are indistinguishable from those seen in patients with larger deletions, indicating that this region may be fundamental to the phenotype. ${ }^{7-10}$ Here we describe the cases of three children who were referred to our hospital with diagnoses of heart disease and no signs of 22q11DS, which turned out to be familial cases of 22q11DS.

\section{Clinical reports}

This study was approved by the ethics committee of the Federal University of Goiás in Goiania, Brazil. Written informed consent were obtained from all family members included in the study.

Proband 1 was a 16-month-old girl born via cesarean section at term. The parents were nonconsanguineous, including a 23-year-old gravida 1, para 1 (G1P1) mother and a 27-year-old father. Her birth weight was 3,280 $\mathrm{g}$ (25-50 $0^{\text {th }}$ percentile), length was $46 \mathrm{~cm}$ ( $3^{\text {rd }}$ percentile), and occipital frontal head circumference (OFC) was $35 \mathrm{~cm}$ $\left(>50^{\text {th }}\right.$ percentile). A clinical examination at the age of 16 months showed a round face, narrow forehead, TOF, and normal development. Clinical examination of the mother revealed a long face, mid-face hypoplasia, retrognathia, slender hands and digits, long toes, hypernasal speech, a speech delay, and a learning disability. The clinical examination of her grandmother showed epicanthic folds, retrognathia, normal

\begin{tabular}{lc} 
Table 1 - Frequency of clinical features in patients with \\
22q11.2DS \\
\hline Clinical features & Frequency \\
\hline Abnormal ears & $45 \%$ \\
Hypoplastic alae nasi & $29.40 \%$ \\
Long face & $41 \%$ \\
High nasal bridge & $19.60 \%$ \\
Broad nasal root/nose & $24 \%$ \\
Micro/retrognathia & $47.70 \%$ \\
Epicanthic folds & $19 \%$ \\
Malar flattening & $32 \%$ \\
Long digits & $68.30 \%$ \\
Narrow palpebral fissures & $73.20 \%$ \\
Hypoplastic face & $34 \%$ \\
Speech delay & $76.40 \%$ \\
Learning disabilities & $75 \%$ \\
Behavioral disturbances & $72.50 \%$ \\
Palatal abnormality & $66.00 \%$ \\
Congenital heart disease & $64.60 \%$ \\
$\quad$ Ventricular septal defect & $35.8 \%$ \\
\hline \multicolumn{1}{l}{ Atrial septal defect } & $28.3 \%$ \\
\hline
\end{tabular}

development, and a surgically corrected cleft palate. Further information from the family also revealed an individual who died at birth due to heart disease (Figure 1A1). Pictures of this family were not allowed for publication.

Proband 2 was a 19-month-old boy who was born via cesarean section at term. The parents were nonconsanguineous, including a 26-year-old G1P1 mother and a 29-year-old father. His birth weight was $3,360 \mathrm{~g}\left(25-50^{\text {th }}\right.$ percentile), length was $47 \mathrm{~cm}$ ( $3^{\text {rd }}$ percentile), and OFC was $35 \mathrm{~cm}\left(>50^{\text {th }}\right.$ percentile). Clinical examination at 19 months of age showed interventricular communication and normal development, with no further clinical features. Clinical examination of his mother revealed a long face, small mouth, dysmorphic 
A

।

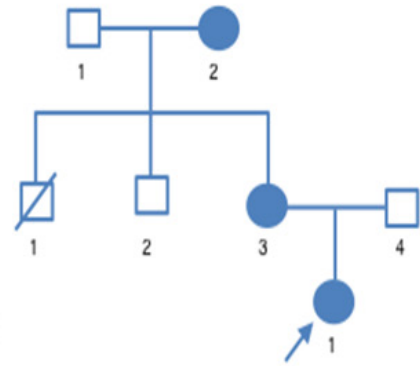

C

I

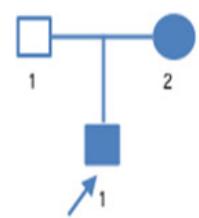

B

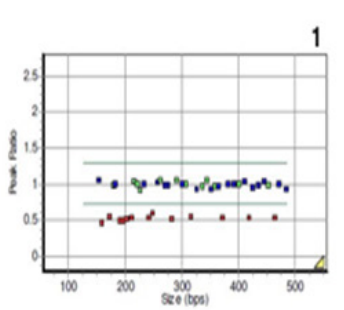

2

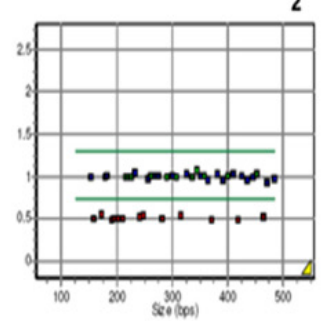

3

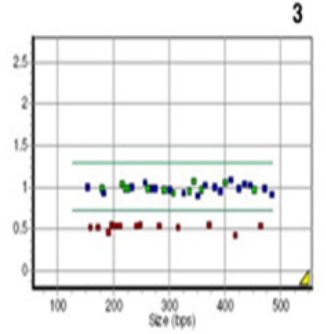

2
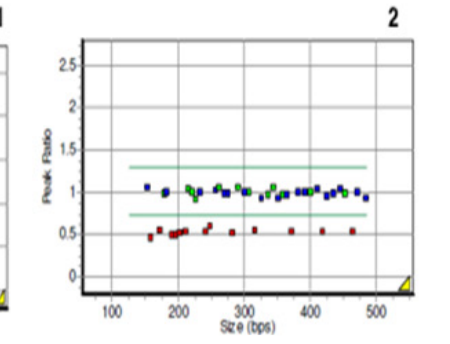

E

$\mathbf{F}$

D

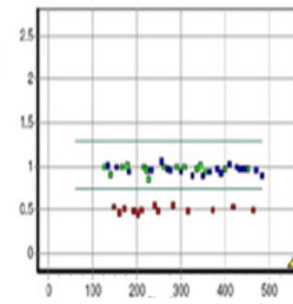

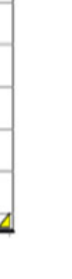

1

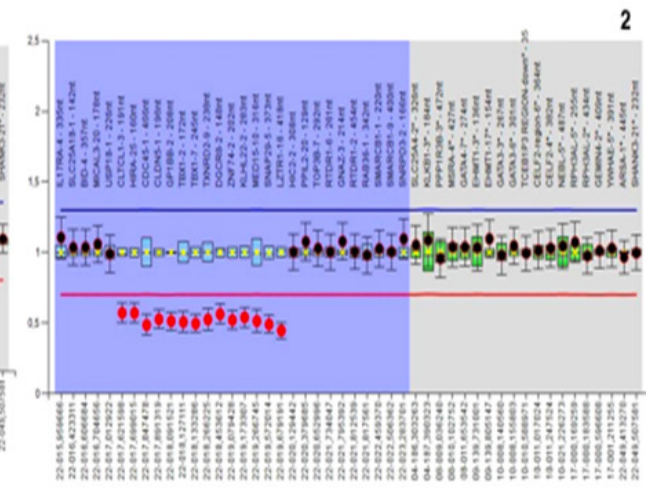

2

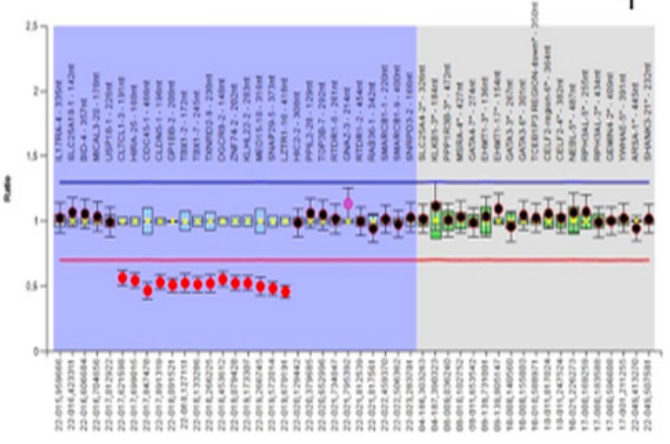

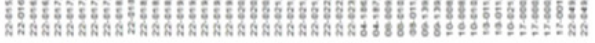

Figure 1 - A. Proband 1' family pedigree. Individuals 1 and 3 presented $3 \mathrm{Mb}$ deletions at 22q11 without congenital heart defects (CHDs). Individual 4 is the proband and presented a $3 \mathrm{Mb}$ deletion at 22q11 with a CHD. Individual 2 had a CHD and died at birth, with no further information. B. Results of multiplex ligation-dependent probe amplification (MLPA) of the proband 1's family showing a deletion from the LCR22-A to LCR22-D in the child (1), mother (2), and grandmother (3). C. Proband 2's family pedigree. Individuals 1 and 2 presented $3 \mathrm{Mb}$ deletions at 22q11, but only individual $2 \mathrm{had}$ a CHD. D. The MLPA results of the proband 2's family showed a deletion from the LCR22-A to LCR22-D in the child (1) and mother (2). E. Proband 3's family pedigree. Individuals 1 and 2 presented $3 \mathrm{Mb}$ deletions at 22q11, both had CHDs. F. The MLPA results of the family of the proband 3 showed a deletion from the LCR22-A to LCR22-D in the child (1) and mother (2).

ears, and learning disability; clinical examination of his father was normal.

Proband 3 was a 2-year-old boy who was born via cesarean section at term. The parents were nonconsanguineous, including a 31-year-old G1P1 mother and a 30-year-old father. His birth weight was $3,120 \mathrm{~g}\left(25-50^{\text {th }}\right.$ percentile), length was $46 \mathrm{~cm}$ ( $3^{\text {rd }}$ percentile), and $\mathrm{OFC}$ was $36 \mathrm{~cm}$ $\left(>50^{\text {th }}\right.$ percentile). His clinical examination at 25 months of age showed pulmonary atresia with interatrial communication and a short stature. Clinical examination of his mother showed upslanting palpebral fissures, a prominent nose, and speech delay. Clinical examination of the father was normal.

\section{Methods}

Multiplex ligation-dependent probe amplification (MLPA) was performed on DNA from peripheral blood 
lymphocytes, extracted using the Puregene Blood Core Kit C (Qiagen Sciences Inc., Germantown, MD, USA). The SALSA MLPA probemix P250 DiGeorge syndrome test kit (MRC-Holland BV, Amsterdam, Netherlands) was used to determine the copy number changes in the 22q11.2 region. The MLPA was performed following the manufacturer's instructions, and all runs included DNA from three normal controls to calibrate the unknown samples. The reaction products were detected using an ABI-3500 Genetic Analyzer (Applied Biosystems Inc., Foster City, CA, USA). To size the polymerase chain reaction (PCR) products and obtain the peak areas, we used the GeneMapper Software (Applied Biosystems Inc.). These data were exported into the GeneMarker software (Softgenetics LLC, State College, PA, USA) or Coffalyser.Net software (MRC-Holland BV) for analysis.

\section{Results}

The MLPA results of the proband 1's family showed a deletion of $3 \mathrm{Mb}$ extending from LCR22-A to LCR22-D in the patient, mother, and grandmother. The MLPA results of the probands 2 and 3's families showed a deletion of $3 \mathrm{Mb}$ extending from LCR22-A to LCR22-D in the patient and mother. Figure 1 shows the family pedigrees of probands 1, 2, and 3, as well as the MLPA results of these families.

\section{Discussion}

CHDs often occur in association with other malformations and as a feature of well-defined genetic syndromes. Frequently, heart defect is one of the first signs of a genetic disorder that may result in an important medical problem in early childhood. ${ }^{11}$ The 22 q11 chromosomal region deletion is considered the second most common cause of CHDs after Down syndrome, ${ }^{12}$ and cardiovascular manifestations of the 22q11 deletion are highly variable. ${ }^{13}$ According to the literature, the del22q11.2 is usually sporadic, with prevalence of familial cases ranging from $6 \%$ to $28 \%$ of patients with this disorder..$^{8-10}$ In the present work, we reported three familial cases of individuals who presented the same 3 $\mathrm{Mb}$ deletion in the $22 \mathrm{q} 11$ region. Approximately $87 \%$ of the patients with 22q11DS have a common $3 \mathrm{Mb}$ deletion region, known as the "common" deleted region (CDR), which includes at least 48 known genes. Smaller deletions may occur more frequently in familial cases than in non-familial cases with del22q11.2. ${ }^{6}$ It has been hypothesized that individuals with the small deletion may have a milder phenotype, and a better chance to produce offspring. ${ }^{13}$ However, molecular analysis of the 22q11.2 region in our families revealed the presence of $3 \mathrm{Mb}$ deletion in an individual that we had considered as having a mild phenotype. Once familial cases are relatively less frequent, it is difficult to affirm that the size of the deletion is related to familial cases or to the phenotype. The expected $90 \%$ frequency of the $3 \mathrm{Mb}$ deletion was observed in the families studied. They presented a clinical variability ranging from the typical characteristics of a 22q11 deletion, as observed in the mother, to only a CHD, as observed in the child.

In the present familial reports, we observed that the affected parent was the mother. Devriendt et al., ${ }^{6}$ reported that the affected parent of all index patients was also the mother. They concluded that this was compatible with the previous hypothesis that this preference for maternal inheritance in familial cases could be due to either decreased fertility ${ }^{13,14}$ or decreased reproductive success ${ }^{13-15}$ in the affected males, with respect to the affected females. However, Matsuoka et al., ${ }^{16}$ suggested that there was no relationship between fertility and del22q11.2. Although the three families has been investigated to fertility, it is possible that there is a preference for maternal inheritance in familial cases, as pointed out by several authors. ${ }^{13,14}$

The role of the $22 \mathrm{q} 11$ region genes in nonsyndromic CHDs is unclear. A mutational analysis of the TBX1 gene, which maps to the 22q11 chromosomal region commonly deleted in patients with DiGeorge/velocardiofacial syndrome, failed to detect the pathogenetic mutations in nonsyndromic individuals with the specific conotruncal defect subtypes commonly found in del22. ${ }^{17,18}$ In the present study, based on the clinical features of the probands, we would not suspect 22q11DS, because the main clinical characteristic was CHD, which was the reason why these children were referred to our clinic. The presence of some of the characteristics of 22q11DS were observed in the mothers (we did not have permission to publish the images of the families), which encouraged us to investigate the presence of the deletion in these individuals. The region in the patients reported here encompasses the TBX1 gene, which can be considered the causative agent, and the differences existing between the mother's and child's phenotypes could be attributed to the presence or absence of genes in the breaking points, plus copy number variations in the rest of the genome. ${ }^{5}$ Moreover, the possibility that 
this intrafamilial phenotype variability may be related to an unknown molecular mechanism or stochastic factors cannot be denied. ${ }^{19}$

Even though the 22q11DS is considered a relatively common chromosomal abnormality, it is still an underdiagnosed condition in the general population, even more so in developing countries and diverse populations, and in many cases the 22q11DS is secondary to a CHD. ${ }^{20}$ In many countries, this conditions have not been systematically recognized by pediatricians, neonatologists and cardiologists, particularly in the first year of life..$^{21}$

The prevalence of 22q11.2DS in patients with CHD has been estimated in attempt to establish a screening for this condition in CHD patients, with or without other features of the syndrome.2,13 In the present study, we investigated three probands that presented CHDs and we identified some of the clinical signs in the mothers, which led us to confirm the presence of 22q11 familial deletions.

The 22q11DS is a constitutional disease with a broad spectrum of phenotypes. Thus, if a child is initially evaluated for CHD, it is probable that other clinical signs will appear throughout the course of the disease. Therefore, the early diagnosis of the syndrome allows a better prognosis and treatment of these patients. It is of note, however, that the presence of microdeletion does not imply the occurrence of postoperative complications.

In the presence of a few or even unique features, as in CHD, within the spectrum of some known syndromes, a familial examination could provide definitive clues for a diagnosis, as well as to prevention and genetic counseling of patients.

\section{References}

1. Pierpont ME, Basson CT, Benson DW, Gelb BD, Giglia TM, Goldmuntz E., et al. Genetic basis for congenital heart defects: current knowledge: a scientific statement from the american heart association congenital cardiac defects committee, council on cardiovascular disease in the young: endorsed by the american academy of pediatrics. Circulation. 2007;115(23):3015-38.

2. Wonkam A, Toko R, Chelo D, Tekendo-Ngongang C, Kingue S, Dahoun S. The 22q11.2 Deletion Syndrome in Congenital Heart Defects: Prevalence of Microdeletion Syndrome in Cameroon. Glob Heart. 2017; 12(2):115-20.

3. Goldmuntz E, Clark BJ, Mitchell LE, Jawad AF, Cuneo BF, Reed L, et al. Frequency of 22q11 deletions in patients with conotruncal defects. J Am Coll Cardiol. 1998; 32(2):492-8.

4. Fernández L, Lapunzina P, Pajares IL, Palomares M, Martínez I, Fernández $\mathrm{B}$, et al. Unrelated chromosomal anomalies found in patients with suspected 22q11.2 deletion. Am J Med Genet A. 2008;146A(9):1134-41.

\section{Author contributions}

Conception and design of the research: Santos MVPF, Ribeiro-Bicudo LA. Acquisition of data: Santos MVPF, Gamba BF, Empke SLL, Alves CC. Analysis and interpretation of the data: Santos MVPF. Gamba BF. Writing of the manuscript : Santos MVPF, Gamba BF, Bérgamo NA. Critical revision of the manuscript for intellectual content: Ribeiro-Bicudo LA.

\section{Potential Conflict of Interest}

No potential conflict of interest relevant to this article was reported.

\section{Sources of Funding}

This study was funded by CAPES and FAPEG.

\section{Study Association}

This article is part of the thesis of master submitted by Bruno Faulin Gamba, from Universidade Federal de Goiás.

\section{Ethics approval and consent to participate}

This study was approved by the Ethics Committee of the CONEP under the protocol number 1.966.673. All the procedures in this study were in accordance with the 1975 Helsinki Declaration, updated in 2013. Informed consent was obtained from all participants included in the study.

5. Shprintzen RJ. Velo-cardio-facial syndrome: 30 years of study. Dev Disabil Res Rev. 2008;14(1):3-10.

6. Devriendt K, Fryns J P, Mortier G, van Thienen MN, Keymolen K. The annual incidence of DiGeorge/velocardiofacial syndrome. J Med Genet. 1998;35(9):789-90.

7. Ganji H, Salehi M, Sedghi M, Abdali H, Nouri N, Sadri L, et al Investigation of TBX1 gene deletion in Iranian children with 22q11.2 deletion syndrome: correlation with conotruncal heart defects. Heart Asia. 2013; 5(1):200-2.

8. Bittel DC, Yu S, Newkirk H, Kibiryeva N, Holt A, Butler MG, et al Refining the 22q11.2 deletion breakpoints in DiGeorge syndrome by aCGH. Cytogenet Genome Res. 2009;124(2):113-20.

9. Fernández L1, Lapunzina P, Pajares IL, Criado GR, García-Guereta $\mathrm{L}$, Pérez J,et al. Higher frequency of uncommon 1.5-2 Mb deletions 
found in familial cases of 22q11.2 deletion syndrome. Am J Med Genet A.2005;136(1):71-5.

10. Maynard TM, Haskell GT, Lieberman JA, LaMantia AS. 22q11 DS: genomic mechanisms and gene function in DiGeorge/velocardiofacial syndrome. Int J Dev Neurosci. 2002; 20(3-5):407-19.

11. McDonald-McGinn D M, Sullivan KE, Marino B, Philip N, zackai E, Emanuel BS, et al. 22q11.2 deletion syndrome. Nat Rev Dis Primers; 2015;1:150-71.

12. Bassett A S, McDonald-McGinn D M, Devriendt K, Digilio M C, Goldenberg P, APractical guidelines for managing patients with 22q11.2 deletion syndrome. J Pediatr. 2011;159(2):332-9.

13. Huber J, Peres V C, de Castro A L, dos Santos T J, da Fontoura Beltrão L. de Baumont A C, et al. Molecular screening for 22Q11.2 deletion syndrome in patients with congenital heart disease. Pediatr Cardiol. 2014;35(8):1356-62.

14. Carlson C, Sirotkin H, Pandita R, Goldberg R, McKie J, Wadey R, et al. Molecular definition of 22q11 deletions in 151 velo-cardio-facial syndrome patients. Am J Hum Genet. 1997;61(3):620-9.

15. Wilson DI, Goodship JA, Burn J, Cross, IE, Scambler PJ. Deletions within chromosome 22q11 in familial congenital heart disease. Lancet. 1992;340(8819):573-5.
16. Matsuoka R, Kimura M, Scambler PJ, Morrow BE, Imamura S, Minoshima S, et al. Molecular and clinical study of 183 patients with conotruncal anomaly face syndrome. Human Genetics. 1998;103(1):70-80.

17. Demczuk S, Levy A, Aubry M, Croquette M F, Philip N, Prieur M, et al. Excess of deletions of maternal origin in the DiGeorge/velo-cardio-facial syndromes. A study of 22 new patients and review of the literature. Hum Genet. 1995;96(1):9-13

18. Iascone MR, Vittorini S, Sacchelli M, Spadoni I, Simi P, Giusti S. Molecular characterization of 22q11 deletion in a three-generation family with maternal transmission. Am J Med Genet. 2002;108(4):319-21.

19. Leana-Cox J, Pangkanon S, Eanet KR, Curtin MS, Wulfsberg EA. Familial DiGeorge/velocardiofacial syndrome with deletions of chromosome area 22q11.2: report of five families with a review of the literature. Am J Med Genet. 1996;65(4):309-16.

20. Conti E, Grifone N, Sarkozy A, Tandoi C, Marino B, Digilio MC, et al. DiGeorge subtypes of nonsyndromic conotruncal defects: evidence against a major role of TBX1 gene. Eur J Hum Genet . 2003;11(4):349-51.

21. Gong W, Gottlieb S, Collins J, Blescia A, Dietz H, Goldmuntz E, et al. Mutation analysis of TBX1 in non-deleted patients with features of DGS/ VCFS or isolated cardiovascular defects. J Med Genet. 2001; 38(12):E45. 